\title{
Application of proteomics in lab diagnosis
}

\begin{abstract}
Proteomics is defined as a large-scale study of proteins, in particular their functions and structures. This review was aimed to introduce the application of proteomics in lab diagnosis. Beforehand, we introduce the methods, which were used in proteomics also the advantages and disadvantages of proteomics are challenged. In the end, the necessity of proteomics for understanding the structure, function, and interaction of proteins in different fields of sciences including biomarkers, drug discovery, etc. will be discussed.
\end{abstract}

Keyword: 2DE; Biomarker; Drug discovery; Protein; Proteomics. 